University of Nebraska - Lincoln

DigitalCommons@University of Nebraska - Lincoln

$1-1998$

\title{
Stress and structure of Ni monolayers on W(110): The importance of lattice mismatch
}

D. Sander

Max-Planck-Institut für Mikrostrukturphysik

C. Schmidthals

Max-Planck-Institut für Mikrostrukturphysik

Axel Enders

University of Nebraska-Lincoln, a.enders@me.com

J. Kirschner

Max-Planck-Institut für Mikrostrukturphysik

Follow this and additional works at: https://digitalcommons.unl.edu/physicsenders

Part of the Physics Commons

Sander, D.; Schmidthals, C.; Enders, Axel; and Kirschner, J., "Stress and structure of Ni monolayers on W(110): The importance of lattice mismatch" (1998). Axel Enders Publications. 3.

https://digitalcommons.unl.edu/physicsenders/3

This Article is brought to you for free and open access by the Research Papers in Physics and Astronomy at DigitalCommons@University of Nebraska - Lincoln. It has been accepted for inclusion in Axel Enders Publications by an authorized administrator of DigitalCommons@University of Nebraska - Lincoln. 


\title{
Stress and structure of Ni monolayers on W(110): The importance of lattice mismatch
}

\author{
D. Sander, C. Schmidthals, A. Enders, and J. Kirschner \\ Max-Planck-Institut für Mikrostrukturphysik, Weinberg 2, D-06120 Halle, Germany
}

(Received 29 September 1997)

\begin{abstract}
The combination of in situ stress measurements, low-energy electron diffraction, and scanning tunneling microscopy reveals the intimate relation between film structure and film stress for epitaxial growth of $\mathrm{Ni}$ on $\mathrm{W}(110)$ in the monolayer range. In contradiction to lattice mismatch considerations, we measure tremendous compressive stress in the pseudomorphic Ni film, where tensile film stress is expected from strain arguments. Surface stress of the film-substrate composite is proposed to be much more relevant for the description of film stress in the submonolayer range than lattice mismatch arguments are. [S0163-1829(98)04603-7]
\end{abstract}

One of the fundamental issues governing heteroepitaxial growth is how the lattice mismatch between film and substrate drives structural modifications in the growing film. ${ }^{1}$ Many structural and morphological changes during the growth of heteroepitaxial systems have been attributed to the lattice mismatch between film and substrate. Structural changes like the formation of misfit dislocation networks ${ }^{2}$ and the change of in-plane atomic positions from pseudomorphic to coincidence structures ${ }^{3}$ have been ascribed to the same driving force, namely, the reduction of the elastic energy density in the growing film. Equally appropriate is the description of the driving force in terms of mechanical stress in the heteroepitaxial system. The reason is that stress is defined as the derivative of the elastic energy density with respect to strain and thus stress tells us directly whether to increase or to lower the atomic density in certain directions to minimize the elastic energy. Many of the structural changes occur in the early stages of heteroepitaxial growth at coverages in the monolayer range. Therefore, in situ stress measurements with submonolayer sensitivity allow a direct examination of the concept of stress as a driving force for structural transitions in the monolayer range. In spite of the fundamental issues related to stress during heteroepitaxial growth, experimental determinations of film stress with submonolayer sensitivity are rare. $^{4-6}$ In this paper we present direct experimental evidence for the intimate relation between film stress and structural transitions for the growth of $\mathrm{Ni}$ on $\mathrm{W}(110)$ from the combination of low-energy electron diffraction (LEED), scanning tunneling microscopy (STM), and stress measurements. For coverages below 0.5 ML we measure considerable compressive film stress in the pseudomorphic Ni film while, based on lattice mismatch arguments, tensile stress is expected. In an extension of existing models for heteroepitaxial growth we suggest that adsorbate-induced modifications of the electronic structure at the film substrate interface dominate the formation of stress in the submonolayer range. For coverages above $0.5 \mathrm{ML}$ our results support the idea of stress as a driving force for structural transitions from pseudomorphic growth to coincidence structures in the monolayer film.

Film stress can be measured with submonolayer sensitivity with optical ${ }^{4}$ and capacitive ${ }^{5}$ methods. Recently, even the tunnel junction of a STM was used to measure surface stress effects in an electrochemical cell. ${ }^{6}$ All these experiments are performed in a way to ensure film growth or adsorption only at the front surface of a thin substrate, whereas the backside remains unaffected. We measure film stress during growth with an optical bending beam technique, which we described previously. ${ }^{4}$ In short, a laser beam is reflected from the bottom end of a $15 \mathrm{~mm}$ long, $3 \mathrm{~mm}$ wide, and $0.12 \mathrm{~mm}$ thin $\mathrm{W}(110)$ crystal, which is clamped to a sample manipulator at its top end. The reflected beam is picked up by a position sensitive detector, thus changes in the radius of curvature due to stress produced by adsorption processes on the front side of the crystal, lead to a deflection of the reflected beam on a position-sensitive detector. Positive (negative) position signals indicate tensile (compressive) stress on the crystal frontside along $\mathrm{W}[001]$.

Direct experimental proof for the intimate relation between film stress and structural changes in the $\mathrm{Ni}$ film is presented in our stress measurement of Fig. 1(a). The stress curve can be plotted versus $\mathrm{Ni} \mathrm{ML}$, as we calibrated the evaporator with a quartz microbalance. After opening the $\mathrm{Ni}$ evaporator, huge compressive stress reaching $-1.3 \mathrm{~N} / \mathrm{m}$ at a deposition of $0.5 \mathrm{ML} \mathrm{Ni}$ is observed. The measured compressive stress is contrary to the tensile stress expected from lattice mismatch arguments. Converted to stress per monolayer, the maximum compressive stress equals an astonishing $13 \mathrm{GPa}$. Stress measurements done during interrupted growth confirmed that the stress values along the curve can be taken directly as stress versus coverage data. Neither relaxation nor thermal effects due to the Ni evaporator affect the measurements. In situ LEED allows us to correlate the various structural phases of the Ni film ${ }^{7}$ indicated in Fig. 1(a) with the respective sections of the stress versus coverage curve. The compressive stress of the pseudomorphic $1 \times 1$ structure is followed by a tensile stress during the formation of the $8 \times 1$ coincidence structure. Further deposition leads to a second minimum of the stress curve at a coverage of $1 \mathrm{ML}$, where the Ni films shows a $7 \times 1$ coincidence structure. The growth of the fcc-like second layer induces tensile stress. No further stress increase is observed up to $8 \mathrm{ML}$ coverage, where tensile film stress sets in. STM revealed that starting from $8 \mathrm{ML}$ on, the growth mode changes from layer by layer to three dimensional. Three-dimensional growth is often found to induce a tensile island interaction, as discussed in detail by Koch. ${ }^{8}$ In the following, we explain first that the different film structures are correlated with different strain 


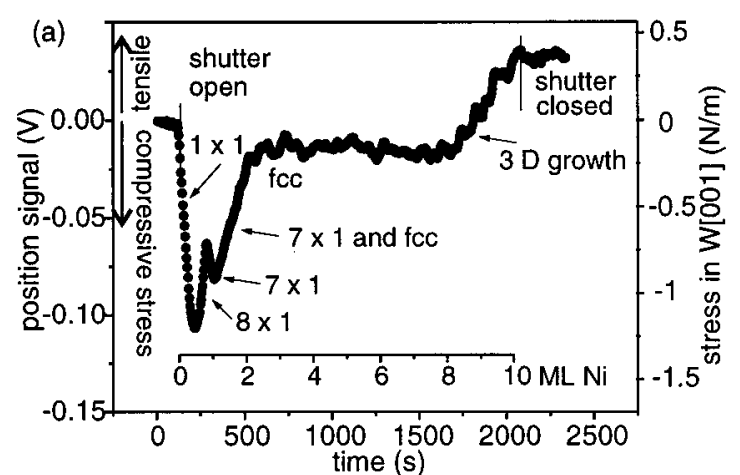

(b) $1 \times 1$ pseudomorphic structure, strain $27 \%$

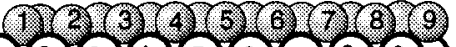
(1)(2) (4) (5) (6) (7)( 8$)$

(c) $8 \times 1$ coincidence structure, strain $13 \%$

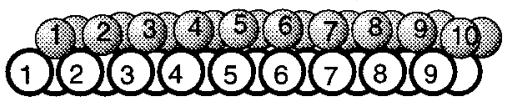

(d) $7 \times 1$ coincidence structure, strain $-1.3 \%$

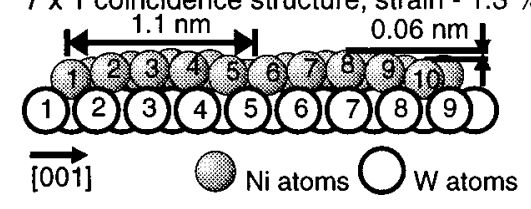

FIG. 1. (a) Stress measurement during the deposition of $10 \mathrm{ML}$ $\mathrm{Ni}$ on $\mathrm{W}(110)$ at $300 \mathrm{~K}$. The arrows indicate the respective film structures at the different $\mathrm{Ni}$ coverages, as determined with LEED and STM. (b)-(d) Hard ball models of the corresponding film structures with calculated strain values. Note, a height corrugation with a repetition length of $1.1 \mathrm{~nm}$ for the $7 \times 1$ structure in (d) [to be seen in Fig. 2(c)].

states within the film, visualized by the hard ball models of Figs. 1(b)-1(d). Then we demonstrate that in the submonolayer range the measured compressive stress cannot be explained by the tensile strain of the pseudomorphic phase. Here, surface stress effects are proposed to be mainly responsible for the measured film stress.

The growth of $\mathrm{Ni}$ on $\mathrm{W}(110)$ proceeds in the NishiyamaWassermann orientation. ${ }^{9}$ In the Nishiyama-Wassermann growth mode, the $\mathrm{Ni}[\overline{1} 10]$ direction is oriented parallel to the $\mathrm{W}[001]$ direction, and $\mathrm{Ni}\left[\frac{1}{2} \frac{1}{2} 1\right]$ is parallel to $\mathrm{W}[\overline{1} 10]$. Along the $\mathrm{W}[001]$ direction the $\mathrm{Ni}$ atoms are separated by a distance $a_{\mathrm{W}}=3.165 \AA$, along $\mathrm{W}[\overline{1} 10]$ the separation is $\sqrt{2} a_{\mathrm{W}}$ $=4.47 \AA$. Throughout this paper, we calculate the strain in a $\mathrm{Ni}-\mathrm{Ni}$ bond with respect to the Ni-Ni separations in a bulk $\mathrm{Ni}(111)$ layer. Along $\mathrm{W}[001]$ a tremendous strain of $\epsilon$ $=\left(a_{\mathrm{W}}-a_{\mathrm{Ni}} / \sqrt{2}\right) /\left(a_{\mathrm{Ni}} / \sqrt{2}\right)=27 \%$ results with $a_{\mathrm{Ni}}$ $=3.524 \AA$, whereas along $\mathrm{W}[\overline{1} 10]$ the strain amounts to moderate $\epsilon=\left(\sqrt{2} a_{\mathrm{W}}-\sqrt{\frac{3}{2}} a_{\mathrm{Ni}}\right) /\left(\sqrt{\frac{3}{2}} a_{\mathrm{Ni}}\right)=3.7 \%$. Based on arguments of epitaxial growth, ${ }^{1}$ a strain in the percent range is generally expected to be tolerable for pseudomorphic growth, as the respective strain energy is not large enough to overcome the energy cost to produce misfit distortions or misfit dislocations in the growing film. However, the strain of almost $30 \%$ induces structural changes in the growing film already in the first layer, as indicated in our stress, LEED, and STM investigations of Figs. 1(a), 2(b), and 2(c), respectively.
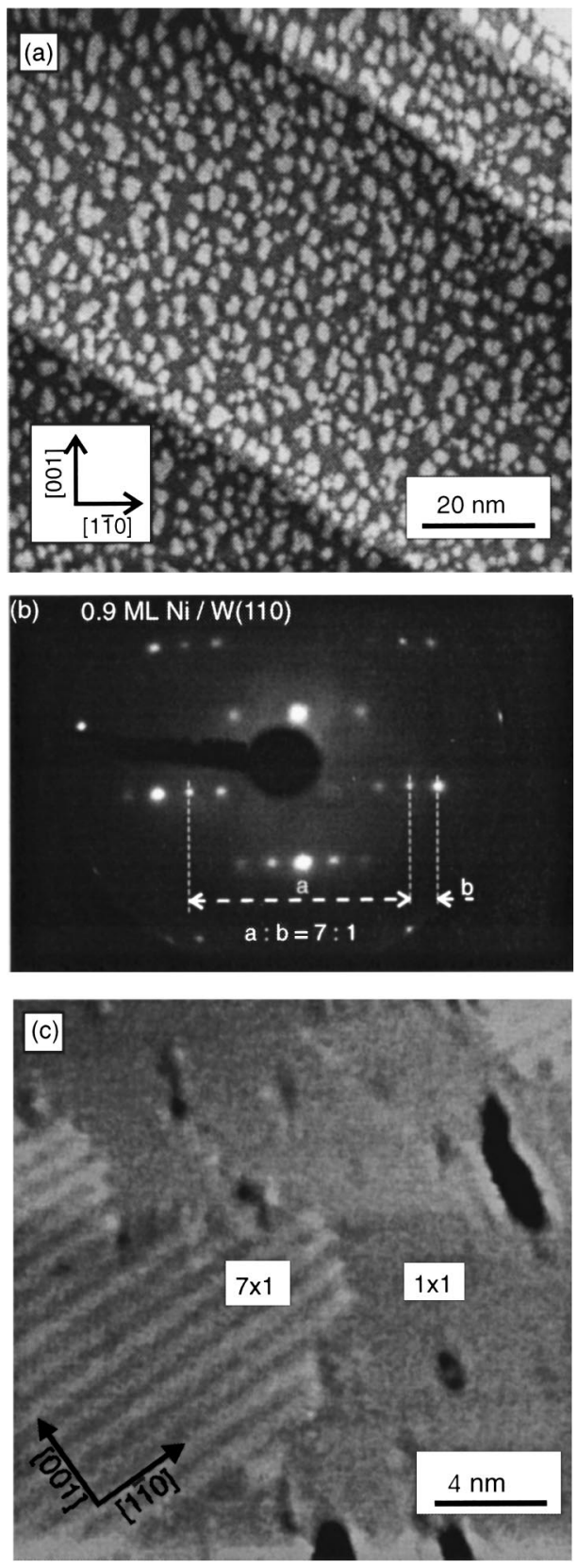

FIG. 2. (a) STM image of 0.4 ML Ni deposited on W(110) at $300 \mathrm{~K}$. Lighter gray Ni islands are shown on the darker W substrate. The stripes running from the upper left to the lower right side are monoatomic steps of the substrate. (b) LEED image of 0.9 ML Ni taken at $129 \mathrm{eV}$. The 7:1 ratio of the spot separation along $\mathrm{W}[001]$ is indicated. (c) STM image taken at a coverage of $0.9 \mathrm{ML}$. The stripe pattern on the lower left side is ascribed to the $7 \times 1$ coincidence structure. The remaining area is assigned to the pseudomorphic $1 \times 1$ structure, openings in the first layer down to the substrate appear as black holes.

At the onset of epitaxial growth of $\mathrm{Ni}$ on $\mathrm{W}(110)$ the $\mathrm{Ni}$ atoms in the pseudomorphic layer are too far away from each other compared to the atomic separation within a $\mathrm{Ni}(111)$ plane. Therefore, based on lattice mismatch arguments, tensile film stress is expected in the pseudomorphic submonolayer range. In complete contradiction to the tensile stress expected from the calculated strain of $27 \%$ shown in Fig. 1(b), our stress measurements of Fig. 1(a) reveal strong com- 
TABLE I. Calculated strain $\epsilon$ in $\mathrm{W}[001], \tau_{\text {calc }}$ calculated and $\tau_{\text {meas }}$ measured stress in $\mathrm{N} / \mathrm{m}$ per ML, $\Delta E$ elastic energy per film atom as calculated in $\mathrm{eV}$, and areal density $n$ of $\mathrm{Ni}$ on $\mathrm{W}(110)$ in $10^{15} \mathrm{~cm}^{-2}$.

\begin{tabular}{lcrrrc}
\hline \hline Ni structure & $\epsilon$ & $\tau_{\text {calc }}$ & $\tau_{\text {meas }}$ & $\Delta E$ & $n$ \\
\hline $1 \times 1$ & 0.27 & 10.5 & -2.9 & 0.695 & 1.41 \\
$8 \times 1$ & 0.13 & 5.5 & 1.9 & 0.176 & 1.59 \\
$7 \times 1$ & -0.013 & 0.4 & -0.8 & 0.011 & 1.82 \\
$\mathrm{fcc}(111)$ & 0 & & & & 1.86 \\
\hline \hline
\end{tabular}

pressive stress along $\mathrm{W}[001]$. We interpret this result as a first hint towards the diminished importance of strain contributions in the submonolayer range. Additional evidence comes from the isotropic island shape in the pseudomorphic growth regime shown in the STM image of Fig. 2(a). We favor the inclusion of surface stress effects to explain the compressive stress, as explained in more detail below. The relations between strain, calculated, and measured film stress, strain energy per film atom, and atomic density for the different Ni structures are summarized in Table I. We calculate film stress and elastic energy per $\mathrm{Ni}$ atom from the elastic energy density ${ }^{10}$ based on bulk elastic constants of $\mathrm{Ni}$. The deficiency of continuum elasticity based on bulk reference data for coverages smaller than $0.5 \mathrm{ML}$ is apparent from Table I. Compressive stress is measured, whereas tensile stress is calculated for $\mathrm{Ni}$ in the submonolayer range. In comparing calculations with the experimental findings, an error bar of $\pm 15 \%$ for the absolute value of the measured stress due to uncertainties in the geometric factors has to be admitted.

With increasing Ni coverage the stress curve of Fig. 1(a) shows, that after a minimum of the stress curve at $0.5 \mathrm{ML}$, tensile film stress sets in. In situ LEED reveals an $8 \times 1$ structure in this coverage range between 0.5 and $0.7 \mathrm{ML}$. A detailed STM and LEED investigation on the dependence of the apparent coverage as seen with STM on the amount of Ni deposited leads us to the interpretation of the $8 \times 1$ LEED pattern in terms of a $8 \times 1$ coincidence structure. As depicted in Fig. 1(c), nine atomic distances in the Ni film equal eight $\mathrm{W}$ atomic distances. The strain is lowered from $27 \%$ of the pseudomorphic range down to $13 \%$ of the $8 \times 1$ structure. The Ni film is still strained, but, as quoted in Table I, the atomic density of the $8 \times 1$ structure is considerably higher with respect to the pseudomorphic phase due to the reduced strain along $\mathrm{W}[001]$. We propose the increase of the atomic density and the resulting lowering of the strain energy compared to the pseudomorphic phase to be the main driving force for the pseudomorphic to $8 \times 1$ transition. The tensile stress measured for the evolving $8 \times 1$ structure for coverages above $0.5 \mathrm{ML}$ reflects the tensile strain of $13 \%$ of that structure. Note, however, the quantitative discrepancy between experiment and our stress calculation summarized in Table I. The slope of the measured curve amounts to $1.9 \mathrm{~N} / \mathrm{m}$ per ML, whereas our simple stress calculation predicts tensile stress of order $5.5 \mathrm{~N} / \mathrm{m}$ per ML, clearly indicating the inappropriateness of the stress calculation for the description of monolayer stress. At best, qualitative description of the slope of the stress curve is obtained for coverages above 0.5 ML for the $8 \times 1$ structure of $\mathrm{Ni}$ with our simple elasticity model. Further Ni deposition leads to the formation of a second stress minimum at $1 \mathrm{ML}$. At that coverage, LEED shows the $7 \times 1$ structure of Fig. 2(b). Again, a combined LEED and STM investigation results in a coincidence model such that nine Ni-Ni distances equal seven $\mathrm{W}-\mathrm{W}$ distances. Thus, a slight compressive strain of $-1.3 \%$ results, as shown in Fig. 1(d). The compressive stress measured for coverages between 0.7 and $1 \mathrm{ML}$ is ascribed to the slight compression of the $\mathrm{Ni}$ atoms in the $7 \times 1$ structure. The $7 \times 1$ diffraction pattern shown in Fig. 2(b) indicates that large areas of the film undergo the structural change, no $8 \times 1$ patterns remain visible. The STM image of Fig. 2(c) shows the coexistence of $1 \times 1$ and $7 \times 1$ areas at a coverage of $0.9 \mathrm{ML}$. The $7 \times 1$ phase is assigned to the stripe pattern in the lower left side of the image. The stripes are oriented along $\mathrm{W}[\overline{1} 10]$ and are separated along $\mathrm{W}[001]$ by $1.1 \mathrm{~nm}$. The simple hard ball model as shown in Fig. 1(d) explains qualitatively the height corrugation visible in the STM image of the $7 \times 1$ coincidence structure. A waviness of the $\mathrm{Ni}$ film with a period of $1.1 \mathrm{~nm}$ results in the $\mathrm{W}[001]$ direction, as visible in the STM image of Fig. 2(c). STM images with larger scan areas indicate that almost $90 \%$ of the first layer show the stripe pattern of the $7 \times 1$ structure. The remaining areas of the first layer are stabilized as pseudomorphic $1 \times 1$ structures on narrow terraces with extensions along $\mathrm{W}[001]$ smaller than $30 \mathrm{~nm}$ and in the vicinity of defectlike holes, as shown in Fig. 2(c). The driving force for the structural transition from an $8 \times 1$ to an $7 \times 1$ structure is ascribed to the further increase of the $\mathrm{Ni}$ atomic density within the first layer and to a reduction of the strain energy by more than a factor of 10 compared to the $8 \times 1$ structure, as shown in Table I. Note that this $7 \times 1$ structure is equivalent to a slightly distorted fcc(111) plane as the $\mathrm{Ni}$ atoms move into intraplanar bonding sites that resemble almost a dense fcc(111) layer, as quoted in Table I.

While the stress measured for thicknesses above 0.5 ML can be explained qualitatively in a simple strain-stress model, the evolution of compressive stress at small coverages is at variance with this model. The atomic picture of pseudomorphic growth in the submonolayer range leads to a highly anisotropic strain within the $\mathrm{Ni}$ islands. Based on strain arguments, an extension of $\mathrm{Ni}$ islands along $\mathrm{W}[001]$ costs 50 times more strain energy compared to extended islands along $\mathrm{W}[110]$, due to a factor 7.5 larger strain along $\mathrm{W}[001]$. In spite of the anisotropic strain, the STM image of Fig. 2(a) shows essentially isotropic Ni islands at a coverage of 0.4 ML. Preferential island orientation is not found before the second layer. There, we ascribe the elongation of the $\mathrm{Ni}$ islands along $\mathrm{W}[001]$ to the smaller strain of $-1.3 \%$ along that direction as compared to $3.7 \%$ strain along $\mathrm{W}[\overline{1} 10]$. Assuming that the island shape is not exclusively determined by kinetic processes, and that the influence of kinetic growth factors are not too different for growth in the first and second layer, we conclude that only in the second layer the island shape can be explained by strain arguments. We interpret the isotropic island shape together with the compressive stress for coverages below $0.5 \mathrm{ML}$ as indications for the inadequacy of interatomic distances of bulk $\mathrm{Ni}$ as a reference scale for the calculation of strain in the submonolayer range.

The high adsorption energy of almost $5 \mathrm{eV} /$ atom of the first monolayer Ni (Ref. 7) suggests a strong electronic Ni-W interaction. A strongly modified electronic structure of 
monolayer $\mathrm{Ni}$ with respect to bulk $\mathrm{Ni}$ results, as shown in photoemission spectroscopy. ${ }^{11}$ To what extent the electronic characteristics of the submonolayer range induce a change of the equilibrium atomic spacing in the Ni layer or in the elastic properties goes beyond the scope of this paper and remains to be investigated. It was reasoned ${ }^{11}$ that for Ni coverages in excess of five atomic layers the formation of a bulklike $d$ band is completed. Assuming that within the $\mathrm{Ni}$ film the bonds are predominantly due to electrons from the Ni $3 d$ and $4 s$ bands, ${ }^{12}$ a bulklike electronic $d$-band structure is therefore certainly a necessary condition for the application of bulk atomic distances and elastic constants in the discussion of Ni film stress. Based on these arguments and the results of stress measurements in the (sub)monolayer range, we conclude that continuum elasticity relying on bulk reference data is of questionable relevance for films thinner than $10 \AA$.

We propose that the inclusion of adsorbate-induced surface stress effects is essential to understand film stress in the submonolayer range. It is well known from theoretical work of different groups that clean metal surfaces are expected to be under tensile stress. ${ }^{13}$ These calculations show that the so-called excess surface stress, which is the strain derivative of the surface free energy, ${ }^{14}$ is of same order as the surface free energy. Thus, the tensile stress of the clean metal surface can be understood as a tendency of the surface layer to contract. In stress measurements, the absolute value of the surface stress cannot be determined, but its change due to adsorption is measured. Therefore, instead of strain in the growing layer, it is the sum of the surface free energy and its strain derivative of the adsorbate-substrate system, the absolute surface stress, ${ }^{6}$ which governs the stress in the submonolayer range. Applying this approach to the interpretation of our stress measurements, it is the difference between the ab- solute surface stress of the adsorbate covered and the clean substrate that determines the resulting stress in the nearsurface region. To gain deeper insight into the atomic origin of stress at interfaces it is mandatory to know how electronic charge between adsorbate and substrate is shifted in the adsorption process. As proposed in a recent $a b$ initio study on excess surface stress, ${ }^{14}$ it has to be considered how the occupation of bonding, nonbonding, and antibonding states is changed due to the charge transfer accompanying the adsorption. In that picture, compressive surface stress can result from a relative shift of charge from bonding to antibonding states, thereby releasing the repulsive Coulomb ion core interaction as compressive stress. Note, that a surface stress of $1 \mathrm{~N} / \mathrm{m}$ translates into an adsorbate-induced change of the surface energy of order tenths of an $\mathrm{eV}$ per surface atom, clearly resembling a significant factor in the substrate-film energetics. Not only for $\mathrm{Ni}$, but also for $\mathrm{Fe},{ }^{15} \mathrm{Co}$, and $\mathrm{Cu}$ on $\mathrm{W}(110)$ we measured compressive stress in the pseudomorphic range where tensile stress is expected from strain arguments. Therefore, the application of a general concept like surface stress in the combined system-substrate surface plus adsorbate atoms-seems promising to explain the stress behavior in the submonolayer range.

In conclusion, we found that submonolayer film stress cannot be adequately described in terms of lattice mismatch and strain energies. We propose to replace the concept of calculating stress from lattice mismatch in the submonolayer range by the analysis of the surface stress of the substratefilm composite to calculate stress. For larger overlayer thickness the lattice mismatch arguments resume validity from a certain coverage on. For Ni on W(110) our stress measurements indicate a borderline of $50 \%$ of one pseudomorphic layer.
${ }^{1}$ J. H. van der Merwe, D. L. Tönsing, and P. M. Stoop, Surf. Sci. 312, 387 (1994); J. H. van der Merwe, J. Woltersdorf, and W. A. Jesser, Mater. Sci. Eng. 81, 1 (1986); I. V. Markov, Crystal Growth for Beginners (World Scientific, Singapore, 1995), Chap. 4.

${ }^{2}$ J. W. Matthews and A. E. Blakeslee, J. Cryst. Growth 27, 118 (1974)

${ }^{3}$ J. H. van der Merwe, E. Bauer, D. L. Tönsing, and P. M. Stoop, Phys. Rev. B 49, 2127 (1994).

${ }^{4}$ A. J. Schell-Sorokin and R. M. Tromp, Phys. Rev. Lett. 64, 1039 (1990); R. E. Martinez, W. A. Augustyniak, and J. A. Golovchenko, ibid. 64, 1035 (1990); J. F. Geisz, T. F. Kuech, M. G. Lagally, F. Cardone, and R. M. Potemski, J. Appl. Phys. 75, 1530 (1994); D. Sander, A. Enders, and J. Kirschner, Rev. Sci. Instrum. 66, 4734 (1995).

${ }^{5}$ M. Weber, R. Koch, and K. H. Rieder, Phys. Rev. Lett. 73, 1166 (1994); D. Sander and H. Ibach, Phys. Rev. B 43, 4263 (1991).

${ }^{6}$ H. Ibach, C. E. Bach, M. Giesen, and A. Grossmann, Surf. Sci. 375, 107 (1997); C. E. Bach, M. Giesen, and H. Ibach, Phys. Rev. Lett. 78, 4225 (1997).

${ }^{7}$ J. Kolackiewicz and E. Bauer, Surf. Sci. 144, 495 (1984).
${ }^{8}$ R. Koch, J. Phys.: Condens. Matter 6, 9519 (1994); (unpublished).

${ }^{9}$ J. H. van der Merwe, Philos. Mag. A 45, 145 (1982); E. Bauer, Appl. Surf. Sci. 11/12, 479 (1982).

${ }^{10}$ P. M. Marcus and F. Jona, Phys. Rev. B 51, 5263 (1995); P. M. Marcus, Surf. Sci. 366, 219 (1996).

${ }^{11}$ K. P. Kämper, W. Schmitt, G. Güntherodt, and H. Kuhlenbeck, Phys. Rev. B 38, 9451 (1988); K. P. Kämper, W. Schmitt, D. A. Wesner, and G. Güntherrodt, Appl. Phys. A: Solids Surf. 49, 573 (1989); C. Koziol, G. Lilienkamp, and E. Bauer, Phys. Rev. B 41, 3364 (1990).

${ }^{12}$ W. A. Harrison, Solid State Theory (Dover, New York, 1980), p. 94.

${ }^{13}$ G. J. Ackland and M. W. Finnis, Philos. Mag. A 54, 301 (1986); R. J. Needs, Phys. Rev. Lett. 58, 53 (1987); M. C. Payne, N. Roberts, R. J. Needs, M. Needles, and J. D. Joannopoulos, Surf. Sci. 211/212, 1 (1989); D. Wolf, ibid. 70, 627 (1993); P. J. Feibelman, Phys. Rev. B 51, 17867 (1995).

${ }^{14}$ V. Foirentino, M. Methfessel, and M. Scheffler, Phys. Rev. Lett. 71, 1051 (1993).

${ }^{15}$ D. Sander, R. Skomski, C. Schmidthals, A. Enders, and J. Kirschner, Phys. Rev. Lett. 77, 2566 (1996). 